\title{
Feasibility of monitoring patient based health outcomes in a routine hospital setting
}

Danny Ruta, Angela Coutts, Mona Abdalla, John Masson, Elizabeth Russell, Peter Brunt, Alistair McKinlay, Ashley Mowat, Thomas Sinclair

\begin{abstract}
Objective-To assess the feasibility of monitoring health outcomes in a routine hospital setting and the value of feedback of outcomes data to clinicians by using the SF 36 health survey questionnaire.

Design-Administration of the questionnaire at baseline and three months, with analysis and interpretation of health status data after adjustments for sociodemographic variables and in conjunction with clinical data. Exploration of usefulness of outcomes data to clinicians through feedback discussion sessions and by an evaluation questionnaire.

Setting-One gastroenterology outpatient department in Aberdeen Royal Hospitals Trust, Scotland.
\end{abstract}

Patients-All (573) patients attending the department during one month (April 1993). Main measures-Ability to obtain patient based outcomes data and requisite clinical information and feed it back to the clinicians in a useful and accessible form. Results-Questionnaires were completed by $542(95 \%)$ patients at baseline and $450(87 \%)$ patients at follow up. Baseline health status data and health outcomes data for the eight different aspects of health were analysed for individual patients, key groups of patients, and the total recruited patient population. Significant differences were shown between patients and the general population and between different groups of patients, and in health status over time. After adjustment for differences in sociodemography and main diagnosis patients with particularly poor scores were identified and discussed. Clinicians judged that this type of assessment could be useful for individual patients if the results were available at the time of consultation or for a well defined group of patients if used as part of a clinical trial.

Conclusions-Monitoring routine outcomes is feasible and instruments to achieve this, such as the SF 36 questionnaire, have potential value in an outpatient setting.

Implications-If data on outcomes are to provide a basis for clinical and managerial decision making, information systems will be required to collect, analyse, interpret, and feed it back regularly and in good time.

(Quality in Health Care 1995;4:161-165)
Keywords: SF 36 health survey questionnaire, patient based health outcomes, clinical management

\section{Introduction}

The government has responded to the need for efficient provision of health care by introducing managed competition into the health service. ${ }^{1}$ Yet without information which relates costs and processes of care to the outcome achieved, it is difficult to evaluate the effectiveness of interventions and to allocate scarce National Health Service resources efficiently. Thus there is an urgent need to incorporate into routine practice measures of outcome that are valid; reliable; responsive to clinically significant changes over time; and, above all, quick and easy to administer in a busy clinical setting. Few outcome measures currently available for routine use satisfy these criteria. In recent years, however, the short form (SF 36) health survey questionnaire has come under close scrutiny for its validity, reliability, and acceptability as a generic measure of health status in both general population ${ }^{2}$ and patient populations. $^{34}$ The SF 36 questionnaire uses eight health scales to measure three aspects of health: functional status, wellbeing, and "overall evaluation of health" (table 1). In routine clinical settings in the United States it was of prognostic value in identifying patients at greater risk of adverse outcomes and those more likely to need focused or further treatment. $^{5-7}$

We describe a study in which the SF 36 questionnaire was used to investigate the feasibility of monitoring health outcomes in a busy hospital outpatient department. The aims of the study were as follows.

- To determine the kind of system necessary to collect health status data and monitor changes in health over time

- To analyse the data appropriately

- To feed this information back to clinicians in a relevant and accessible manner.

Table 1 SF 36 health profile

\begin{tabular}{lr}
\hline Health attributes and concepts & $\begin{array}{c}\text { No } \\
\text { items }\end{array}$ \\
\hline I Functional status: & \\
(a) Physical functioning & 10 \\
(b) Social functioning & 2 \\
(c) Role limitations attributed to physical problems & 4 \\
(d) Role limitations attributed to emotional problems & 3 \\
II Wellbeing & \\
(a) Mental health & 5 \\
(b) Energy and fatigue & 5 \\
(c) Pain & 2 \\
III Overall evaluation of health & \\
(a) General health perception & 5 \\
\hline
\end{tabular}




\section{Methods}

SUBJECTS AND SETTING

The project was set in the gastroenterology outpatient department of Aberdeen Royal Hospitals Trust which, as the only district general hospital for north east Scotland, receives all types of gastroenterology patients for a population of 0.5 million people. On average there were five consultation sessions and over 100 patients a week, covering the full range of gastrointestinal diagnoses. Patients who attended during one month (April 1993) were followed up three months later; their clinical management in the interviewing period ranged from discharge to general practitioner care to inpatient admission and surgery. Patients with more than one appointment in April were counted only on the first occasion.

During the initial phase of the project the research staff and four participating consultant gastroenterologists (PB, AK, AM, TS) met several times to discuss possible outcomes criteria, the clinical requirements of a data collection system, and the most appropriate setting and timing of administration of the questionnaire. After this initial planning phase a study design was agreed.

STUDY DESIGN

Feasibility of data collection

Preliminary investigations were undertaken by the researcher on the rate of patient throughput, the "normal" waiting time per patient, and the suitability of the patients' waiting room as an appropriate setting. Possible times at which patients might practically be given the SF 36 questionnaire to complete were discussed. After a trial session it was considered feasible that the questionnaires should be given by the researcher (AC) to the patients before they saw the doctor. Therefore, during April 1993, all patients attending a clinic in the gastroenterology outpatient department in Aberdeen were asked to complete a questionnaire in the clinic waiting area before seeing the doctor. The questionnaire comprised an anglicised version of the SF 36 questionnaire and sociodemographic questions. The responses were taken as the baseline data. All patients were sent a follow up postal questionnaire three months after their outpatient appointment. Reminders were sent to non-respondents after two weeks.

A clinical profile comprising the items chosen by the consultant gastroenterologists as those most important in assessing and managing the patient was completed for each patient by the researcher. This included the following clinical data abstracted from the case notes: primary diagnosis and any active comorbid diagnoses, current treatment, previous major surgery, results of laboratory tests, lifestyle details, and inpatient episodes in the past year. The clinical profiles were subsequently entered on to computer for analysis. Data on diagnosis and surgery were converted to Read $\operatorname{codes}^{8}$ before data entry, for ease of data handling. Read codes were chosen for direct comparison with any subsequent or similar studies as there was no other agreed local coding system in operation at the time and in the belief that this system was to become nationally operational in the foreseeable future.

\section{Analysis of SF 36 questionnaire results}

The data were analysed to present the gastroenterologists with information on two parameters: individual patients and aggregated patient groups. Firstly we calculated baseline SF 36 scores for each patient. Although the distributions of the scores for some of the SF 36 scales are known to be skewed, regression analysis has been shown to be a valid means of analysing SF 36 data. ${ }^{4}$ Multiple regression ${ }^{9}$ was therefore used to estimate, for each patient, "predicted" SF 36 scores across the eight scales - that is, the scores predicted for a patient by the regression model, taking account of the patient's age, sex, socioeconomic status, and primary diagnosis. Ninety five per cent confidence intervals were estimated for the predicted scores. ${ }^{9}$ The regression analysis also permitted identification of "outliers" whose actual scores deviated more than three standard deviations from their predicted scores. A similar analysis was then used to estimate, for each patient, the expected change in health status at three months across the eight SF 36 scales, based on the change in scores of other patients with the same characteristics.

Secondly, to assess the value of health status information in describing the health characteristics of different groups within a patient population adjusted mean SF 36 scores at baseline and three months were calculated for several different groups. These included patients grouped by primary diagnosis or presenting complaint, whether they were new patients or returning patients, their sex, and whether they were a recent inpatient. The clinicians initially selected the groups during the planning phase, although they identified additional groups after reviewing the preliminary results. The mean SF 36 scores for patients grouped by primary diagnosis and presenting complaint were compared with each other and with population norms for Aberdeen city. (A previously surveyed random sample of 900 members of the general population, selected from the electoral register for Aberdeen served as a comparison group. ${ }^{4}$ )

For all other group analyses comparisons were made with the total gastroenterological population. Multiple regression was used to calculate these adjusted means, controlling for age; sex; socioeconomic status; and, when appropriate, presenting complaint or diagnosis. The means of the differences in SF 36 group scores at baseline and at three months were also calculated and assessed for significant change over time with the paired $t$ test.

\section{Feedback and evaluation}

Three outcome audit meetings attended by the gastroenterology medical staff were held to determine whether the data provided by the SF 36 questionnaire were likely to help in clinical management or policy decisions. At the first meeting the methods of analysis and mode of presentation of results were explained, and 
Table 2 SF 36 baseline and predicted scores for a man with inflammatory bowel disease

\begin{tabular}{lcc}
\hline Health parameter & $\begin{array}{c}\text { Baseline score } \\
(\%)\end{array}$ & $\begin{array}{c}\text { Predicted score } \\
\text { (95\% confidence interval) }\end{array}$ \\
\hline Physical function & 5 & $79 \cdot 2(32 \cdot 6$ to 100$)$ \\
Social function & 100 & $81 \cdot 6(28 \cdot 0$ to 100$)$ \\
Role limitations: & 0 & $67 \cdot 4(0$ to 100$)$ \\
$\quad$ Physical & 100 & $79 \cdot 6(3.9$ to 100$)$ \\
Emotional & 84 & $75.9(38.9$ to 100$)$ \\
Mental health & 100 & $71 \cdot 8(20 \cdot 8$ to 100$)$ \\
Pain & 35 & $60 \cdot 8(17.6$ to 100$)$ \\
Energy and fatigue & 10 & $62 \cdot 3(18 \cdot 0$ to 100$)$ \\
\hline General health perception
\end{tabular}

^Predicted score compared with all other patients adjusted for age, sex, socioeconomic status, and main diagnosis. The higher the score, the better the health status.

response rates and baseline health status data were presented and discussed. Data were presented for "outliers" for physical function and for another 10 patients who were "outliers" for mental health. The second outcome audit meeting concentrated on the results of requested analyses of data on the groups and on change over time for individual patients. In the final meeting SF 36 scores for groups of patients with the five most common conditions were assessed in detail. These sessions were tape recorded and the data analysed for comments about the comprehensibility of the presentation of the data; clarification of data collection methods; reactions of clinicians to, and discussion of, results; possible repercussions on management decisions; and requests for further data analysis. Together with a semistructured questionnaire administered to the clinicians at the end of the project, the feedback meetings provided the basis for a qualitative evaluation of the clinical value of outcome monitoring in this setting.

\section{Results}

RESPONSE RATES

Baseline data collection covered 23 clinical sessions, during which 666 appointments were scheduled and $573(86 \%)$ patients attended, of whom 542(95\%) completed the SF 36 questionnaire. The mean age of respondents was $48 \cdot 1$ (standard deviation 16.7) years; $10(2 \%)$ were aged under 18 years, and $344(64 \%)$ were female. Patients consulted with over 103 different primary diagnoses. The five most common types of condition (covering $44 \%$ of patients) were ulcerative colitis, Crohn's disease, irritable bowel syndrome, abdominal pain, and gastrooesophageal reflux. At follow up 518 question- naires were posted, of which $450(87 \%)$ were returned. Follow up questionnaires were not posted to patients whose medical notes had not been available for abstraction before the follow up data collection began. At baseline 57 patients $(11 \%)$ returned incomplete SF 36 questionnaires; however, only four $(<1 \%)$ could not be analysed. ${ }^{10}$

\section{MANAGEMENT OF INDIVIDUAL PATIENTS}

The clinicians thought that the SF 36 results gave them a more holistic view of the baseline health status of their patients. We present the results for two patients as examples.

Table 2 shows the SF 36 baseline and "predicted" scores for a 53 year old man with inflammatory bowel disease, who also had parkinsonism, fibrosing alveolitis, otosclerosis and pressure urticaria. (The higher the score the better the health status.) On comparison with his "predicted" scores, adjusted for age, sex, social class, and primary diagnosis, this patient was identified as an outlier for physical function. His score for general health was also well outside the range predicted. Scores for other aspects of health fell within the $95 \%$ confidence interval. For simplicity and because of the variety of comorbid conditions and their low frequency it was not possible to take account of other comorbid conditions in the regression analysis. However, to facilitate the interpretation of the health status information all comorbid conditions recorded in the case notes are listed. For this particular patient, his poor scores for physical function and general health were attributed to his parkinsonism and alveolitis, in addition to his bowel disease.

Data on health outcomes showing change in health status over time were obtained from the analysis of the follow up data. Table 3 shows outcomes data for a 55 year old woman with Crohn's disease, identified as an outlier for mental health. She also had polycystic disease of the kidney and liver, hypertension, and diabetes and had an ileocaecal resection and had taken a drug overdose (date given). The table shows her actual baseline scores with her predicted baseline scores and her scores after three months. In summary, her scores improved in six out of eight aspects of health and were unchanged in the remaining two.

In both of these patients the clinicians were prompted to produce reasonable hypotheses to explain the SF 36 distributions, and were stimulated to seek further clinical information.

Table 3 SF 36 baseline and follow up scores for a woman with Crohn's disease

\begin{tabular}{|c|c|c|c|c|}
\hline \multirow[t]{2}{*}{ Health parameter } & \multicolumn{2}{|c|}{ Baseline score } & \multicolumn{2}{|c|}{ Follow up score } \\
\hline & $\begin{array}{l}\text { Score } \\
(\%)\end{array}$ & $\begin{array}{c}\text { Predicted score } \\
\text { (95\% confidence interval) }\end{array}$ & $\begin{array}{c}\text { Score } \\
(\%)\end{array}$ & $\begin{array}{c}\text { Predicted score } \dagger \\
\text { (95\% confidence interval) }\end{array}$ \\
\hline Physical function & 5 & $61 \cdot 4(14 \cdot 7$ to 100$)$ & 60 & $21.4(0$ to $53 \cdot 8)$ \\
\hline Social function & 0 & $67 \cdot 2(13 \cdot 6$ to 100$)$ & $11 \cdot 1$ & $21 \cdot 7(0$ to $63 \cdot 3)$ \\
\hline \multicolumn{5}{|l|}{ Role limitations: } \\
\hline Physical & 0 & $46 \cdot 8(0$ to 100$)$ & 0 & $11 \cdot 6(0$ to $76 \cdot 9)$ \\
\hline Emotional & 0 & $61 \cdot 7(0$ to 100$)$ & 0 & $17 \cdot 7(0$ to $85 \cdot 1)$ \\
\hline Mental health & 8 & $66(29.0$ to 100$)$ & 12 & $24 \cdot 1(0$ to $51 \cdot 3)$ \\
\hline Pain & $22 \cdot 2$ & $56 \cdot 4(8 \cdot 1$ to 100$)$ & $88 \cdot 9$ & $37.7(0$ to 76.0$)$ \\
\hline Energy and fatigue & 0 & $38 \cdot 7(0$ to $82 \cdot 1)$ & 5 & $12 \cdot 1(0$ to $53 \cdot 8)$ \\
\hline General health perception & 10 & $42 \cdot 4(0$ to $87 \cdot 1)$ & 25 & $12 \cdot 3(0$ to $38 \cdot 5)$ \\
\hline
\end{tabular}

$\star$ Predicted baseline score compared with all other patients adjusted for age, sex, socioeconomic status and main diagnosis. †Predicted follow up score compared with change in all other patients, adjusted for age, sex, socioeconomic status, main diagnosis and baseline score. 


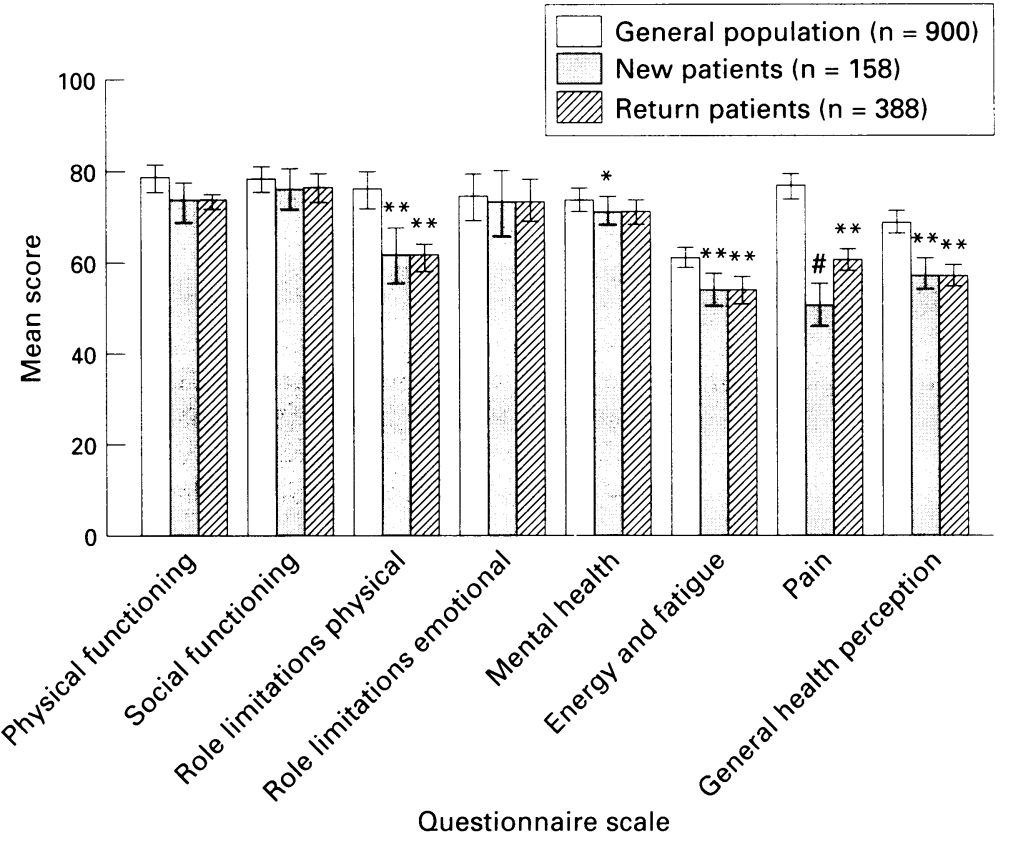

Fig 1 Mean scores at baseline of eight scales of $S F 36$ health profile in new (158) and returning (388) patients attending gastroenterology clinic compared with those of the general population (542) adjusted to age, sex, and socioeconomic status of the general population. Bars are $95 \%$ confidence intervals. Significant score difference between patients and general population: ${ }^{\star} p \leqslant 0.05,{ }^{\star} p \leqslant 0.01 \%, \# p \leqslant 0.001 \%$.

\section{GROUP ANALYSIS}

Figures 1 and 2 shows the use of SF 36 questionnaire for group comparisons. Figure 1 shows the SF 36 scores at baseline for all aspects of health for new (158) and returning (388) patients compared with the general population. Scores for all aspects of health were significantly lower (that is, worse) in gastroenterology patients than in the general population. However, the scores for new and returning patients were not significantly different except for pain, for which they were significantly higher in returning patients $(p \leqslant 0.01 \%)$. This suggests that clinicians are alleviating at least some pain in patients referred to their care.

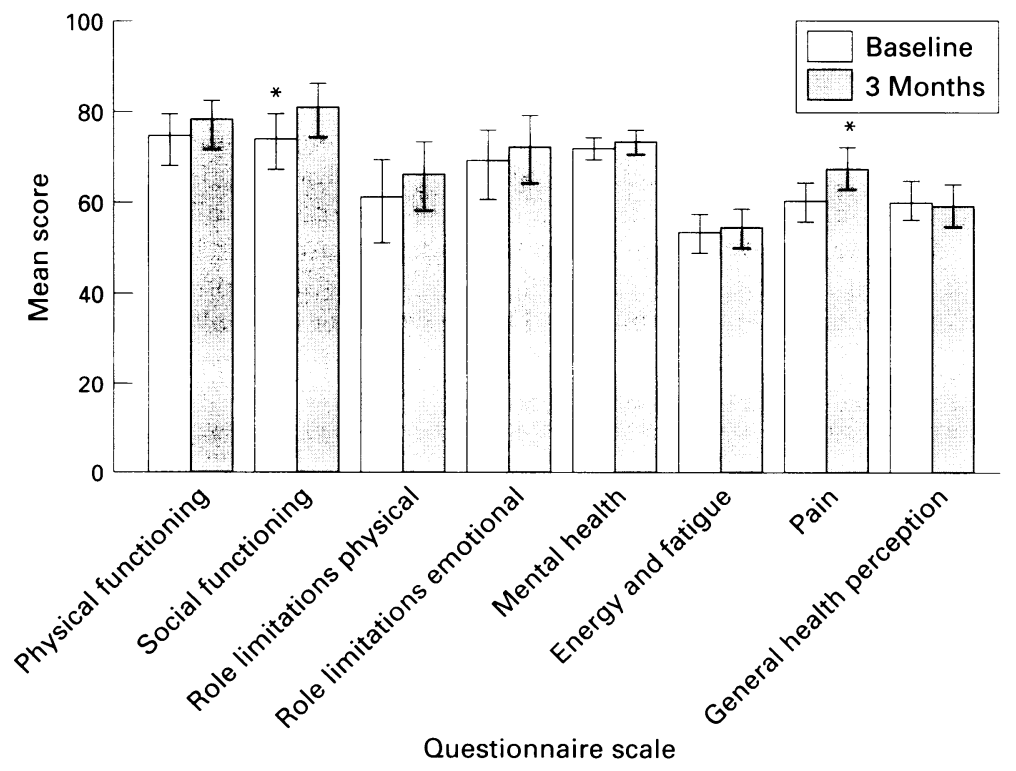

Fig 2 Mean scores of eight scales of SF 36 health profiles for all new patients attending gastroenterology clinic at baseline and three month follow up (117). Bars are $95 \%$ confidence intervals. Difference between baseline and follow up scores $p \leqslant 0 \cdot 1 \%$ (paired t test).
Figure 2 shows the SF 36 data over time for 117 patients who were new at the time of baseline data collection. These scores indicate that their perceived level of social function and pain significantly improved in the three months since first consultation.

VALUE TO CLINICIANS

The clinicians who participated in this study welcomed the in depth assessment of their patients' health made possible by the SF 36 questionnaire. Their attention was drawn to aspects not usually addressed during a gastroenterology consultation. For individual patients the clinicians thought that this kind of measure could provide new insights and pose questions which might not otherwise have arisen in a busy outpatient clinic, especially if the results could be made available at the time of consultation. The results of large groups of patients tended to be difficult to interpret because of the acknowledged problems of case mix differences in disease severity and comorbid conditions. However, the data did begin to provide information suggesting that, particularly for new patients, the process of clinical care resulted, on average, in a favourable health outcome on the two important health dimensions of social functions and pain. The clinicians also expressed the view that the SF 36 questionnaire might provide a useful tool if incorporated as part of clinically controlled trials for well defined groups of patients.

\section{Discussion}

In this study we showed the feasibility of collecting patient based health status data on outpatients routinely attending a busy gastroenterology clinic and of assessing health outcomes after three months. No other data collection system was tested because of time constraints and because the clinical discussions disclosed few alternatives within the clinic setting. Future studies should consider the feasibility and usefulness of data collection from patients at, for example, referral or appointment. Abstracting clinical data from the case notes was laborious and painstaking. However, these data seemed to be helpful, if not essential, to the clinicians' interpretation of the SF 36 results and change in health status over time, and they would have to be included in any future system despite the effort involved. Further work is also required to develop techniques which take account of comorbidity when comparing health outcome between patient groups, treatments, or providers.

In the analysis of patient management the statistical technique of providing 95\% confidence intervals for predicting a patient's score on the SF 36 scales provided very wide confidence intervals for some of the scales. In predicting an observed SF 36 score for a given individual, two sources of error operate: individual error, as measured by the standard deviation of the particular SF 36 scale, and the error of estimating the mean of that individual from the regression equation. The second type of error is likely to be reduced with larger sample size, as the estimation procedure will 
become more precise. As for the first type of error, the standard deviation of some of the SF 36 scales, such as roles of physical and emotional aspects of the disease in daily living, are known to be quite large for either patients or the general population. It is quite unlikely, therefore, that these two scales would be useful in managing individual patients. At least two of the scales - physical function and mental health - seem to be valuable in detecting clinically significant differences between individual patients and the general clinic population. A longer term study with a larger sample size, and hence narrower confidence intervals, is required, however, to determine just how sensitive these scales might be if used routinely.

In this study SF 36 results were available to clinicians only some time after the data collection period because of the time required for abstracting the clinical data from the case notes and subsequent analysis of the SF 36 scores from the database. In some studies in the United States an "Opscan" version of the SF 36 questionnaire, which is fed into a computer in the clinic and analysed within five seconds, allows the doctor to review the results during the patient consultation, when the case notes are at hand. This type of feedback can improve patient care in several ways. For example, in Portland in the United States the SF 36 physical function score and the Harris hip score are presented to hip replacement patients at their pre-operative and postoperative assessment. These patients are also shown a graph indicating the predicted improvement in scores in the year immediately after surgery, based on aggregated scores for patients undergoing the same operation. ${ }^{7}$ This reassured patients about their own recovery, modified their expectations of the outcome of surgery, and thereby eased their frustrations at limitations persisting six months or more after surgery. In another study, in which routinely collected SF 36 data were used to review the care of patients undergoing renal dialysis, ${ }^{5}$ the feedback of information to clinicians prompted them to consider the effect on patient wellbeing of a move toward shorter dialysis sessions and tighter control of weekly blood concentrations of urea.

In conclusion, this study shows the feasibility of routine outcomes monitoring and gives an indication of the potential value of using instruments like the SF 36 questionnaire in an outpatient setting. If such information about health outcomes is to provide a rational basis for clinical and managerial decision making throughout the health service, information systems are required that will routinely collect, analyse, interpret, and feed back data on health outcome for most patients coming into contact with the health service. Larger pilot studies are needed to address the logistical and technical difficulties of developing such information systems and to determine the optimum package of outcome measures required for different clinical conditions and client groups.

This study was supported by a grant from the King's Fund Centre and the Scottish Home and Health Department. We thank Dr Hamish Wilson, Director of Contracts for Grampian
Health Board for his helpful collaboration, and Dean Phillips Health Board for his helpful collaboration, and
and Johann Coutts for computing assistance.

1 Secretaries of State for Health, Wales, Northern Ireland, and Scotland. Working for patients. London: HMSO, 1989. (Cm 555.)

2 Jenkinson C, Coulter A, Wright L. Short form 36 (SF 36) health survey questionnaire: normative data for adults of health survey questionnaire: normative

3 Bardsley MJ, Coles JM. Practical experiences in auditing patient outcomes. Quality in Health Care 1992;1:124-30.

4 Garratt AM, Ruta DA, Abdalla MI, Buckingham JK, Russell IT. The health survey questionnaire: an outcome measure suitable for routine use within the NHS? $B M \mathcal{F}$ 1993;306:1440-4.

5 Kurtin PS, Ross Davies A, Meyer KB, DeGiacomo JM, Kantz ME. Patient-based health status measures in outpatient dialysis: early experiences in developing an outcomes assessment program. Med Care 1992;30(suppl): MS136-49.

6 Nerenz DR, Repasky DP, Whitehouse FW, Kahkonen DM. Ongoing assessment of health status in patients with

7 Lansky D, Butler JBV, Waller FT. Using health status measures in the hospital setting: from acute care to "outcomes management." Med Care 1992;30(suppl):

8 Buckland $R$. The language of health. $B M F$ 1993;306: 287-8.

9 Kleinbaum DG, Kupper LL, Muller KE. Applied regression analysis and other multivariable methods. Boston: PWSKent Publishing, 1988.

10 Wetzler HP, Radosevich D. Interstudy. Health status questionnaire (SF 36) technical report. 1992. 